\title{
Study of Pre-Analytical Errors in a Clinical Biochemistry Laboratory
}

\author{
Dr. Srirekha. $\mathrm{P}^{1}$, Dr. R.S. Swaroopa Rani ${ }^{2}$, Dr. Sarada U ${ }^{3}$, Dr. B. Ravindra Reddy ${ }^{4 *}$ \\ ${ }_{1,3}^{3}$ Assistant Professor, Department of Biochemistry, Kurnool Medical College, Kurnool \\ ${ }^{2}$ Assistant Professor, Department of Biochemistry, Guntur Medical College Guntur \\ ${ }^{4}$ Associate Professor, Department of Biochemistry, SanthiRam Medical College, Nandyal
}

Article History
Received: 06.12 .2021
Accepted: 06.01 .2022
Published: 10.01 .2022
Journal homepage:
https://www.easpublisher.com
Quick Response Code

\begin{abstract}
Background: A diagnostic error was a much wider issue in the Laborotory Medicine and further steps are required towards improving in Understanding the issue to reduce the errors in Lab. The current study about the nature of laboratory testing associated errors mainly concerns from ordering test to interpretation of results and importance in reducing errors. Objective: To study errors in $24 \mathrm{hrs}$ lab of GGH Kurnool for 3 months over the whole testing cycle including pre-analytical, analytical, and post-analytical phases. Materials and methods: The number of different type of errors in Pre- analytical, analytical and post-analytical phases was recorded on designed proforma for 2 months from Oct 2021 to NOV 30 2021. All collected data was entered and analysed by using SPSS version 21. Results: Over 2 months, all venous and arterial blood samples were received in $24 \mathrm{hrs}$ lab of GGH. Of the 1,80, 011 samples received during the study period, 2340 samples were found to be unsuitable for testing, accounting for $1.30 \%$ of the rejection. All these samples were rejected due to different types of preanalytical errors that are due to All these samples were rejected due to different types of pre-analytical errors that are due to misidentification $(0.06 \%)$, incorrect tube $(0.1 \%)$ missing samples $(0.06 \%)$, draw from IV site $(0.09 \%)$, inadequate samples $(0.5 \%)$, wrong timing of sample collection $(0.09 \%)$, hemolysed samples $(0.3 \%)$ and lipemic samples $(0.1 \%)$. Conclusions: The use of a consensuallydefined list of evidence-based Quality indicators to be applied in the accreditation programs of clinical laboratories according to the current International Standard (ISO 15189:2012) is an effective tool for improving quality, decreasing the risk of errors and increasing patient safety.
\end{abstract}

Keywords: Laboratory Errors, Pre-Analytical, Analytical, Post Analytical, Total Testing Process.

Copyright (C) 2022 The Author(s): This is an open-access article distributed under the terms of the Creative Commons Attribution 4.0 International License (CC BY-NC 4.0) which permits unrestricted use, distribution, and reproduction in any medium for non-commercial use provided the original author and source are credited.

\section{INTRODUCTION}

In Hospitals, two-thirds of important clinical decisions on patient treatment, diagnosis, and therapeutic monitoring of most diseases and management are based on laboratory test results. Hence the central Laborotory was Back bone of the hospital. The whole process of testing of a patient's blood from it's ordering to its testing and then to its reporting and ultimately reaching the treating doctor can be divided into three broad steps.

1. Pre-analytical: specimen collection, transport and processing

2. Analytical: testing

3. Post-analytical: testing results transmission, interpretation, follow-up, retesting
Overall the three processes combined are called as 'total testing processes'. The ISO 15189:2008 standard for laboratory accreditation defines the preanalytical phase as 'steps starting in chronological order, from the clinician's request and including the examination requisition, preparation of patients, collection of the primary sample, and transportation to and within the laboratory, and ending when the analytical examination procedure begins. Examination errors include equipment failure, sample mix-ups, interference whereas post-examination errors include systemic review, formatting and interpretation, authorization for release, reporting and transmission of the result and storage of sample of the examination [1]. Pre-analytical error can account up to $70 \%$ of errors during the total diagnostic process [2]. Proper examining and preventing pre-analytical critical errors within clinical laboratories atmost important to reduce 
errors through the thorough finding of any defect within this phase that will have unfavourable impact on the patient safety, treatmet, health service, health workers effort and cost [3]. Moreover, it's essential to identify whether the pre-analytical error depends on a laboratory professional (e.g.calibration) or nonlaboratory personnel (e.g. error in patient identification and/or blood collection. This identification will improve the performance of the clinical laboratories as well as analytical quality by reduction of the turnaround time (TAT), accurate patient identification, effective patient diagnosis, treatment of diseases, clinical monitoring, and disease prevention [4].

Table-1: Shows Laboratory Total Testing Process and Their Potential Errors

\begin{tabular}{|c|c|}
\hline $\begin{array}{c}\text { Pre-Analytical } \\
\text { Phase }\end{array}$ & $\begin{array}{l}\text {-Missed Test Requisition Form (TRF), Incorrect sample identification, } \\
\text { - Incorrect sample tube Sample from IV running area } \\
\text {-Delay in sample transportation Insufficient samples Sample mix-ups Tube broken in centrifuge } \\
\text { Wrong timing for Collection,Invalid Specimen: Haemolysed Sample, Lipemic Sample \& Icteric } \\
\text { Sample }\end{array}$ \\
\hline $\begin{array}{l}\text { Analytical } \\
\text { Phase }\end{array}$ & $\begin{array}{l}\text {-Instrument not calibrated properly,Specimen mix-up. Inadequate specimen, Presence of } \\
\text { interfering substances, Wrong analytical method, Lack of precision,Post-analytical phase, Wrong } \\
\text { patient identification }\end{array}$ \\
\hline $\begin{array}{c}\text { Post-Analytical } \\
\text { Phase }\end{array}$ & $\begin{array}{l}\text {-Wrong patient identification, Report not legible, Report delayed, Transcriptional error,. } \\
\text { Specificity of the test not understood, Previous values are not available for comparison }\end{array}$ \\
\hline
\end{tabular}

\section{Materials ANd Methods}

A prospective study was done for a period of 2 months from $1^{\text {st }}$ OCT 2021 to 30 Nov 2021 in 24 hrs Clinical laboratory, GGH, Kurnool. The ethical clearance was obtained from the Institutional Ethics Committee (IEC), GGH, Kurnool has bed capacity of about 1200 beded which is equipped with clinical biochemistry laboratory. The main analyzers operated here are AU 480 for routine clinical chemistry. Other equipment in the laboratory includes Swelab analyser for electrolyte analysis, Beckman for vitamins, Thyroid function tests \& fertility profiles. The Lab provides routine and special tests and it is a part of Christian Medical College (CMC) Vellore External Quality Assurance Scheme (EQAS) for chemistry, special hormones.

Outpatient samples are collected in the op blood collection chamber by Lab technician and since it is close to the laboratory sample are immediately transported to the laboratory for processing. Samples from inpatient are collected by the nursing staffs and transported to the laboratory through concerned personnel through proper manner taking special precaution were ever needed.

An average of 1, 80, 011 samples were received and observed over the study period of 2 months. Blood samples were collected by $2 \mathrm{ml} \& 5 \mathrm{ml}$ syringe and needle in the plain vaccutainers for routine biochemistry tests, in fluoride for estimation of blood sugar and EDTA for HbA1c test, whereas urine, CSF and other fluid were collected in sterilized containers.
Color coded tubes are used to collect blood; urine and other biological fluid are collected in sterilized plastic collection bottles. Samples from IP are received by lab technician on duty after checking that the samples have been sent in appropriate vials, there is adequate sample volume, blood in anti-coagulants vials have not clotted, vials have been properly labeled and test requisition sheet are properly filled. The time when the sample is received is also noted. The patient sample is then given a number and then sent for sample Processing.

All types of preanalytical errors were recorded systematically. The error rate was calculated as a $\%$ of error observed in total no of samples against total no of samples observed in the lab for the stipulated time, under the following categories: All types of preanalytical errors were recorded systematically under the following categories:

1. Incorrect sample identification: mismatch between the name on sample and test requisition form (TRF)

2. Missed Sample

3. Sample from IV running area

4. Inadequate Sample: sample received is not sufficient for testing

5. Wrong timing for Collection - lipid profile

6. Haemolysed Sample: Presence of pink to red tinge in serum or plasma.

7. Lipemic Sample: Presence of turbidity in serum or plasma caused by the accumulation of lipoprotein particles

8. Inappropriate Transport 
9. Inappropriate centrifugation

10. Patient's not properly prepared for the test.

\section{RESUlTS AND DisCUSSION}

Data were collected from 24 hrs Clinical Biochemistry laboratory GGH Hospital, Kurnool and analyzed by using SPSS version 19 . The study results showed that out of the $1,80,011$ blood samples screened over a period of two months; Oct 2021 to
Nov 2021, pre-analytical errors were observed in 2340 samples which is approximately $1.3 \%$ of the total number of samples received.

Table-1: Main characteristics of the study

\begin{tabular}{|l|l|}
\hline Gender & $\mathbf{N}(\mathbf{\%})$ \\
\hline Female & $68,005(38 \%)$ \\
\hline Male & $1,12,00(62 \%)$ \\
\hline Inpatients & 138223 \\
\hline Out patients & 41796 \\
\hline
\end{tabular}

Table-2: Pre-analytical error for different categories

\begin{tabular}{|l|l|l|l|l|l|l|}
\hline & IP & \% & OP & \% & TOTAL & \% \\
\hline Total Sample & $1,38,223$ & & 41796 & & $1,80,011$ & \\
\hline Misidentification & 56 & 0.04 & 52 & 0.02 & 108 & 0.06 \\
\hline Incorrect tube & 88 & 0.04 & 92 & 0.06 & 180 & 0.1 \\
\hline Missing Samples & 60 & 0.04 & 45 & 0.02 & 108 & 0.06 \\
\hline Draw from IV site & 162 & 0.12 & 0 & 0 & 162 & 0.09 \\
\hline Inadequate Sample & 560 & 0.41 & 340 & 0.1 & 900 & 0.5 \\
\hline Wrong timing of sample collection & 25 & 0.02 & 132 & 0.07 & 162 & 0.09 \\
\hline Hemolysed & 346 & 0.25 & 192 & 0.1 & 540 & 0.3 \\
\hline Lipaemic & 93 & 0.07 & 97 & 0.04 & 180 & 0.1 \\
\hline Total & 1390 & 0.99 & 950 & 0.4 & 2340 & 1.3 \\
\hline
\end{tabular}

All these samples were rejected due to different types of pre-analytical errors that are due to misidentification $(0.06 \%)$, incorrect tube $(0.1 \%)$ missing samples $(0.06 \%)$, draw from IV site $(0.09 \%)$, inadequate samples $(0.5 \%)$, wrong timing of sample collection $(0.09 \%)$, hemolysed samples $(0.3 \%)$ and lipemic samples $(0.1 \%)$.

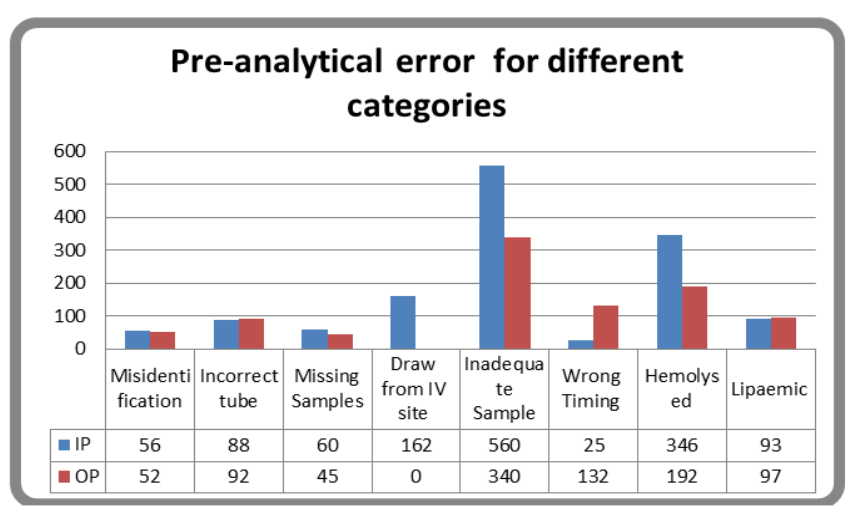

\section{DiscUSSION}

The main scope of our study was to assess the frequency of pre analytical errors, in order to evaluate and to quantify performance in the pre analytical phase of the Total Testing Process.

Clotted samples: Improper sample collection technique, Improper Reagent mixing technique, Incorrect Sample Vacutainer/Tube, Not immediately placing blood into the Vacutainer/tube after drawing blood using syringe, Very slow drawing of blood into a tube. Leads to Clotted sample.it will cause 1. Request for Repeat Sample 2. Delay in Diagnosis and Treatment 3. Increase in Length of stay in Hospital. 4. Increase in related Healthcare cost .control measures include•
Education \& training of healthcare staff responsible for sample collection Closed System of blood collection is practiced only in OPD sample collection room Education \& Training for staff about Hospital Protocol (SOP of sample collection \&Transportation). - Use of sample collection tray* - Use of Closed System of blood collection is recommended in IP wards. • Assessment of the frequency of system errors caused by sample clot [5].

The most common pre-analytical error, $(0.5 \%)$, observed in mainly the Inpatient was insufficient sample volume. Majority of these samples were received from NICU and Pediatric wards. This may be attributed to the fact that it is difficult to collect blood samples in pediatric patients. Lippi and his fellow 
members reported insufficient specimen quantity and quality accounting for over $60 \%$ of pre-analytical errors.

Hemolysis leads to extravasation of intracellular contents in to plasma leading to false high values of potassium and intracellular enzymes such as Acid Phosphatase (ACP), Lactate Dehydrogenase (LDH), Aspartate Transaminase (AST), Alanine Transaminase (ALT), Creatinine, Creatine Kinase (CK), albumin, alkaline phosphatase (ALP), chloride, gammaglutamyl transferase (GGT), glucose and sodium. Parameters like Potassium, Alanine Transaminase (ALT), Creatinine, Creatine Kinase (CK) are overestimated when hemolysed sample are used for analysis, whereas parameters like albumin, alkaline phosphatase (ALP), chloride, gamma-glutamyl transferase (GGT), glucose, bilirubin and sodium are underestimated when hemolysed sample are used for analysis. The various causes for hemolysis found to be Cleansing the venipuncture site with alcohol and not allowing the site to dry appropriately (at least $30 \mathrm{sec}$ ), syringe draws, vigorous mixing of the samples, transferring the sample into a tube by pushing down on the syringe plunger to force blood into a tube \& not allowing the serum specimen to clot for the recommended amount of time can result in fibrin formation in the serum [6]. It also prolongs Turnaround time due to need of fresh sample for processing the request. Insufficient blood volume was another reason for rejection. Every analysis requires a fixed volume of serum /plasma for analysis. The main reasons behind this sampling are ignoranceof the phlebotomist, difficult sampling as in pediatric patients, patients with chronic, debilitating diseasr and patients on chemotheraphy whose the veins are difficult to localize [7].

Lipemic samples accounted for $0.1 \%$ of rejection. Lipemic samples arise due to wrong timing of sample collection (post-meals) and if a patient is diagnosed have hyperlipoproteinemias. This can be avoided by advising for overnight fasting along with post one week treatment overnight sample if it was not emergency and for emergency samples sample dilution method and interference testing assay was performed. In case of patient diagnosed to have hyperlipoproteinemias, we requested the clinicians mention it on TRF. The direction and magnitude of lipemia interference in spectrophotometric assays depends on wavelength of the reaction and blanking of the method. Especially like AST, ALT, glucose which uses NADPH at $340 \mathrm{~nm}$ is affected. Lipemia results in falsely decreased levels of serum electrolytes due to high proportion of lipid in plasma $(25 \%)$ which is normally around $8 \%$ in plasma, hence water portion is only about $75 \%$ compared to $92 \%$ water in normal plasma [8].

In our Study Patient misidentification $0.06 \%$ of total samples .Patient misidentification errors are potentially associated with the worst clinical outcomes because of the possibility of misdiagnosis and mishandled therapy. This may be probably, due to heavy work load and it is important to identify a patient accurately so that blood is collected from the correct person. Drawing blood from the wrong person or labelling the correct patient's sample with a different patient's label can certainly contribute to laboratory error [9].

Icterus or hyperbilirubinemia is the presence of elevated bilirubin levels, occurs due to increased bilirubin production or inappropriate excretion as we see in hemolytic anemia, liver diseases or biliary tract obstruction. Icteric serum or plasma ranges in color from dark yellow to bright yellow, rather than normal straw color. The abnormal colour of the serum can interfere with photometric measurements because of its ability to react chemicals in other reagents resulting in decreased analyte values or spectral interferences during color measurement. Concentrations of bilirubin greater than $2.5 \mathrm{mg} / \mathrm{dL}$ can lead to clinically relevant changes of anti-thrombin. Higher concentrations can interfere with coagulation tests. In our study, were found $1.37 \%$ of icteric samples [10].

The next most common pre-analytical error, $(0.91 \%)$, observed in mainly the Indoor samples (1.74\%) was insufficient sample volume. Only 3 OPD sample volumes were inadequate while in case of indoor samples it was 213. Majority of these samples were received from NICU and Pediatric wards. This may be attributed to the fact that it is difficult to collect blood samples in pediatric patients. Lippi and his fellow members reported insufficient specimen quantity and quality accounting for over $60 \%$ of pre-analytical errors.

Collecting Sample in a wrong tube/ Vacutainer, Using Unsterile container for sample collection, Lack of Knowledge about Vacutainers and its order of draw can increase TAT, Request for Repeat Sample. Misdiagnosis and Wrong Treatment Copy of guidelines for order of draw has to be stick on walls right above the sample collection tray in wards to reduce this type of error[11].

Our study findings were similar to the study done by Bhagya shree et al., Priyanka Prasad et al., Sangeeta Kapoor et al., Iram Hamid et al.

Bhagya shree et al. study concluded that Total errors detected were 1232. The most common error was hemolysed sample. A total of 926 hemolysed samples were received in the 2 years period of study making up $75.16 \%$ of the errors. The next commonly encountered error was of insufficient sample volume, which was $17.53 \%$ of the errors. Other errors contributed $7.31 \%$, of which sample in inappropriate container was $0.57 \%$, delay in sample transport was $1.79 \%$, wrong numbering 
and sample not received was $4.14 \%$, clotted sample was $0.41 \%$ and contamination from infusion route was $0.41 \%$.

Priyanka Prasad et al. concluded that the overall percentage of rejection is $2.9 \%$. $0.92 \%$ was rejected due to hemolysis; $0.58 \%$ was blood collected in wrong tubes; $0.55 \%$ was clotted blood; $0.26 \%$ had inappropriate timing of collection; $0.24 \%$ was mislabeled samples; $0.20 \%$ had insufficient sample quantity and $0.14 \%$ was lipemic samples [13].

Sangeeta Kapoor et al. Study concluded that pre analytical errors in our laboratory were found to be $2.32 \%$ [14]. Iram Hamid et al. study concluded that that pre analytical error in our laboratory was found to be $0.93 \%$ [15].

To overcome preanalytical errors, the following corrective measure have been followed

1. Skilled staff: skilled and adequate staff to maintain collection standards, which give an extra verge of expertise.

2. Phlebotomists: with proper knowledge pertaining to phlebotomy (trained personnel)

3. Regular educational competency assessments should be encouraged to allow (new and old personal) an opportunity to recognize and manage errors.

4. Vacutainers: Proper knowledge regarding use of evacuated tube system to the lab pertaining to sample volume and use of anti-coagulants.

5. Transport: Laboratory Personnel guided regarding importance of Transport of specimen promptly to the laboratory at the earliest after collection to avoid errors to delay.

6. Advanced Technology: Usefulness of barcode scanners system for individual sample recognition [12].

\section{CONCLUSION}

In present study pre-analytical error rates were analysed for the samples received at $24 \mathrm{hrs}$ Clinical Biochemistry laboratory GGH Hospital, Kurnool for the period of Six months.

1. The practice of keeping a record of the errors at all stages of analysis and then devising corrective strategies for their prevention can gradually make laboratory free from such errors.

2. The promotion of ideal phlebotomy practices and sample transport procedures is a pre-requisite for

3. The efficacy of laboratory functioning. All the staff working in the laboratory should be given appropriate training and participate in many continuing medical education programmes
Then devising corrective strategies among different hospital according to the common cause's forrejections for their prevention, which can gradually free a laboratory from such errors.

\section{REFERENCES}

1. Austin, J. (2016). Public Health Epidemiol, 3(5); 1048.

2. Saloni, M. N. (2020). Study of pre analytical errors in clinical biochemistry laboratory in rural area of Punjab. Panacea J Med Sci,10(2);135-138

3. Plebani, M. (2012). Quality indicators to detect preanalytical errors in laboratory testing. Clin Biochem Rev, 33(3); 85-88.

4. Plebani, M. (2013). The CCLM contribution to improvements in quality and patient safety. Clin Chem Lab Med, 51; 39-46.Search in Google Scholar

5. Pfützner, A., Schipper, C., Ramljak, S., Flacke, F., Sieber, J., Forst, T., \& Musholt, P. B. (2013). Evaluation of the effects of insufficient blood volume samples on the performance of blood glucose self-test meters. Journal of diabetes science and technology, 7(6), $1522-1529$.

6. Fidler, J. R. (2007). Task analysis revisited: Refining the phlebotomy technician scope of practice and assessing longitudinal change in competencies. Evaluation \& the health professions, 30(2), 150-169.

7. Koseoglu, M., Hur, A., Atay, A., \& Cuhadar, S. (2011). Effects of hemolysis interference on routine biochemistry parameters. Biochemia medica, 21(1), 79-85.

8. Calmarza, P., \& Cordero, J. (2011). Lipemia interferences in routine clinical biochemical tests. Biochemia medica, 21(2), 160-166.

9. Sushma, B., \& Shrikant, C. (2019). Study on "preanalytical errors in a clinical biochemistry laboratory": the hidden flaws in total testing. Biochem Anal Biochem, 8(374), 2161-1009.

10. Jagannatha, S. B., Chandrakar, S., \& Durg, C. Study of Pre-Analytical Errors in a Clinical Biochemistry Laboratory: The Hidden Flaws in Total Testing.

11. Golla, R., \& Manjunatha, R. (2019). A study on identifying the pre-analytical phase errors leading to biochemistry and hematology sample rejection by using fmea \& paretos principle. Int. J. of Adv. Res. 7 (Sep). 811-820] (ISSN 2320-5407.

12. Lippi, G., Salvagno, G. L., Montagnana, M., Franchini, M., \& Guidi, G. C. (2006). Phlebotomy issues and quality improvement in results of laboratory testing. Clinical laboratory, 52(5-6), 217-230.

13. Kapoor, S., Verma, A., \& Saxena, I. (2014). Preanalytical errors in clinical chemistry laboratory of a tertiary care hospital in western Uttar Pradesh, India. International Journal of Bioinformatics and Biological Science, 2(3and4), 127-133.

14. Kapoor, S., Verma, A., \& Saxena, I. (2014). Preanalytical errors in clinical chemistry laboratory of a tertiary care hospital in western Uttar Pradesh, India. International Journal of Bioinformatics and Biological Science, 2(3and4), 127-133.

15. Hamid, I, Pai, V.R., Raghavendra, U., Sheriff, M.H. (2019). Pre-analytical errors in clinical biochemistry-a comparative study. Int J Clin Biochem Res, 6(2); 182189.

Cite This Article: Dr. Srirekha. P, Dr. R.S. Swaroopa Rani, Dr. Sarada U, Dr. B. Ravindra Reddy (2022). Study of Pre-Analytical Errors in a Clinical Biochemistry Laboratory. East African Scholars J Med Sci, 5(1), 5-9. 